\section{High-entropy alloys: An interview with Jien-Wei Yeh}

\author{
www.mrs.org/fall2016 \\ Interviewed by N. Balasubramanian
}

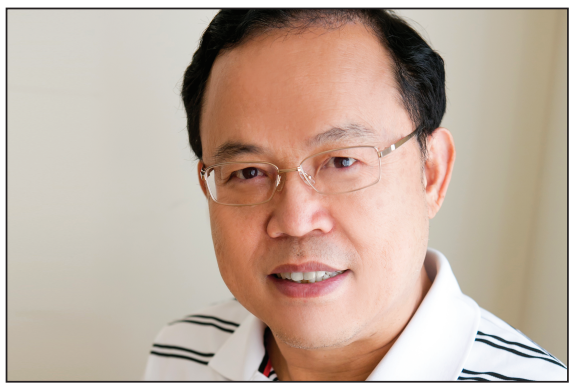

not mix together many different kinds of elements to increase the confusion and complexity and to enhance the chaos? This would allow the alloy to become more homogeneous in composition, possessing good properties and without forming compounds. When I went back to my lab that night, I talked to my graduate student, K.H. Huang, about this concept, and this started our exploration of this unknown world.

The exploration and development of high-entropy alloys was very difficult in the beginning because there were no previous publications. Luckily, the National Science Council of Taiwan, ROC (now the Ministry of Science and Technology) appreciated my projects and provided continued funding since 2001. Later, the Ministry of Economics and Industrial Technology Research Institute of Taiwan also supported the development of applications. By then, I led a team that consisted of my colleagues as well as master's and $\mathrm{PhD}$ students in further exploration and development.

the cost, but also downgraded the origina merits of the fine microstructure and properties of rapidly solidified powders.

After this, I invented a reciprocating extrusion method, in which symmetric double extruders share the same die, to cooperatively hot-extrude a round alloy billet through back and forth movement. Accumulative dynamical recrystallization refines the grain structure and inclusions to the finest level and renders the alloys and composites strong and tough. However, the method is costly when considering the two extruders and cycling time expended in the extrusion.

In 1995, while driving along a countryside road to Taipei, I asked myself: Why
Although I defined high-entropy alloys, filed the first patents, and published early papers, Professor B. Cantor of the UK [now Vice Chancellor of the University of Bradford] and Professor S. Ranganathan of India [Indian Institute of Science] were also looking into multicomponent alloys. Prof. Cantor conducted the preliminary study on equiatomic multicomponent alloys. I regard them also as the pioneers in this field.

\section{How will HEAs impact materials science?}

I divide the alloy world into three categories: high-entropy alloys (with more powder to produce bulk $\mathrm{Al}$ alloys. The 
than five major elements), mediumentropy alloys (with 3 or 4 major elements), and low-entropy alloys (with 1 or 2 major elements). Most conventional alloys are low-entropy alloys, and some conventional concentrated alloys belong to the medium-entropy group. As for ceramics and polymers, the same classification applies when referring to the number of major components. Materials science reports, including physical metallurgy and solid-state physics, are mostly based on the observations of and data from traditional or low-entropy materials. With research on high-entropy alloys and related materials, it could be expected that traditional materials science would have some interactions with the observation and data of the new category.

Research on high-entropy alloys and related materials is important not only for satisfying scientific curiosity, but also in understanding the whole materials world. In addition, there are many research topics in HEAs and related materials, and it is easier to find new topics. Therefore, I always encourage early career materials scientists to broaden their minds and to understand highentropy alloys and related materials.

\section{What applications do you foresee?} Traditional materials have been used successfully in many applications, but we found many critical applications where even the best of them are not satisfactory. For example, cutting tools need improved wear resistance and lifetimes under high speed and/or dry conditions. Either the materials for cutting tools and/or the hard coatings on them should be improved. Turbine engines need to be improved for higher efficiency. Oxidation-resistant and creep-resistant materials for higher operating temperatures are thus needed. Other applications include molds and dies, turbochargers, satellite nozzles, fourth-generation nuclear reactors, room-temperature superconductors, components with a low temperature coefficient of resistance in a large temperature range, non-sticky coatings, 3C (computer, communication, and consumer electronics) housings, golf club heads, oil well bearings, core components of injection molding machines, and propellers and piping in chemical and petrochemical plants.

Some particular applications may be easier than others for industrialization, such as functional coatings, 3C casings, and golf club heads. For example, I have used lost wax mold and ceramic mold (Shaw process) to cast strong and tough high-entropy alloys for high-temperature parts used in hightemperature wear-testing machines and

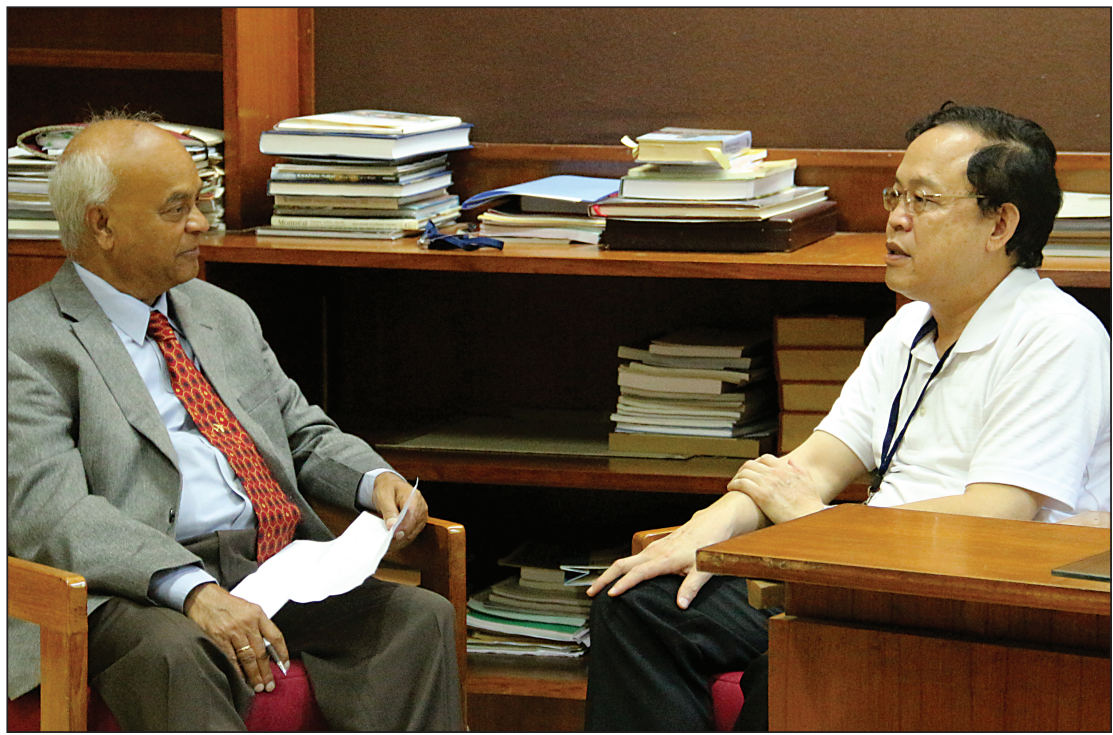

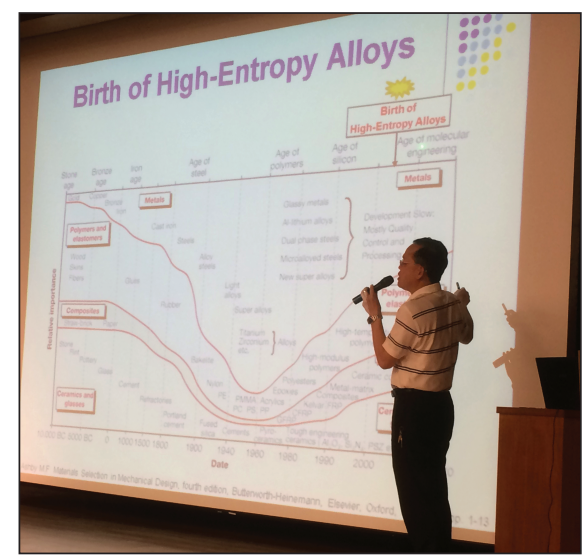

tensile-testing machines. Applications such as aerospace and nuclear reactor materials would be more critical and take time for certification. The bridging between academia and industry is important for assessing and testing promising candidate materials. The lack of bridging would delay industrialization.

This year, I have begun to collaborate with local enterprises in an effort to solve their materials bottlenecks encountered in production processes and products. I am confident of success because my database and those published could provide routes for solutions.

During the research, I not only emphasized academic study but also kept in mind application-oriented assessment and possibility. In this regard, it is helpful for me to be closer to industry.

Jien-Wei Yeh, Yong Zhang (University of Science and Technology, Beijing), P.K. Liaw (University of Tennessee, Knoxville), and Robert O. Ritchie (University of California, Berkeley) are co-organizers of Symposium MB3 on HEAs at the 2016 Materials Research Society Fall Meeting in Boston. Among the invited speakers is HEA pioneer Brian Cantor (University of Bradford, UK). Topics will include new developments as well as advanced applications; mechanical, electrochemical, and physical behaviors; latest technologies for fabrication and characterization; serration behaviors and crackling noise; and fundamental studies and theoretical modeling. 